\title{
Synergistic toxicity and physiological impact of thiamethoxam alone or in binary mixtures with three commonly used insecticides on honeybee
}

\author{
Yufei Wang, Wenxin Zhang, Tengfei Shi, Shengyun Xu, Bozhi Lu, Hongwei QIN, \\ Linsheng $\mathrm{YU}_{\mathrm{U}}$
}

Anhui Agricultural University, Hefei 230036, China

Received 26 November 2018 - Revised 14 October 2019 - Accepted 13 November 2019

\begin{abstract}
As one of the most widely used insecticides, thiamethoxam (Th) plays an important role in agriculture pest control but is hazardous to the health of honeybees (Apis mellifera ligustica). In agricultural practice, several types of insecticides are often applied simultaneously, but their synergistic effects on honeybees remain elusive. Here, we treated honeybees with various binary mixtures of insecticides in which a sublethal dose of Th was mixed with three other common insecticides: $\lambda$-cyhalothrin $(\lambda$-cy), $\beta$-cypermethrin $(\beta$-cy), and abamectin $(\mathrm{Ab})$. The mortality rates of the groups treated with the insecticide mixtures were higher than that obtained with the pesticides alone. Specifically, the Th $+\lambda$-cy and Th $+\mathrm{Ab}$ combinations exerted a synergistic effect $(P<0.05)$ on bees and resulted in significantly higher mortality than that obtained with the single treatments. However, the Th $+\beta$-cy mixture had no synergistic effect $(P>0.05)$ on bees, and the mortality rate of this group was almost identical to those of the groups administered these pesticides alone. Furthermore, the Th $+\lambda$-cy and $\mathrm{Th}+\mathrm{Ab}$ mixtures significantly suppressed the activity and expression of glutathione S-transferases (GSTs), which are key detoxification enzymes in insects. This result suggests that the $\mathrm{Th}+\lambda$-cy and $\mathrm{Th}+\mathrm{Ab}$ combinations at a given concentration adversely affect the survival of honeybees. Our study provides novel information for the selection of pesticide combinations based on protecting honeybee populations.
\end{abstract}

honeybee / insecticide / thiamethoxam / binary mixture / synergistic effect

\section{INTRODUCTION}

Honeybees are important pollinators of crops and wild plants. Due to their specificity for pollination, relocation, and domestication, honeybees are the most ideal pollinators for agricultural production. Bees simultaneously provide various high valueadded products, such as honey, royal jelly, and beeswax. However, bees have recently been affected by a wide range of biological and abiotic factors (Moritz et al. 2010), such as insecticides, pathogens,

Corresponding author: L. Yu, yulinsheng@ahau.edu.cn Handling Editor: Monique Gauthier and parasites (Nazzi and Le Conte 2016), and the separate and combined effects of these factors have led to a significant reduction in the number of bees worldwide (Goulson et al. 2015). This issue should be highlighted and has thus attracted international attention. The abuse of insecticides is probably one reason for the observed reduction in bees. The neonicotinoid insecticides function as nicotinic acetylcholine receptor (nAChR) agonists that selectively bind to nAChRs (Millar \& Denholm 2007). Due to their high affinity to nAChRs and their inability to be degraded by acetylcholinesterase, neonicotinoid insecticides induce continued excitation of the insect's nervous system and block central nervous signal transduction, which eventually leads to death (Elbert et al. 2008; Matsuda et al. 2001). $\lambda$ - 
cyhalothrin and $\beta$-cypermethrin belong to the group of pyrethroid insecticides, which is considered a type of broad-spectrum efficient insecticide whose mode of action is mainly to tag and stomach poison. Pyrethroid insecticides modify the functioning of the voltage-gated ion channels, leading to the interruption of the nerve impulse conduction along the axons. In honeybees, survival and high brain functions like memory are impaired after exposure to lambda-cyhalothrin (Liao et al. 2018). These pesticides are most widely used in agricultural production due to their high efficacy, long-lasting effects, and low toxicity to mammals (Christen et al. 2018).

Due to the large-scale use of these pesticides in the field, the pests can be effectively controlled, but nontarget insects, such as pollinating bees, can also be hurt (Goulson et al. 2015). Based on the residues of neonicotinoid insecticides in the field and the amount of nectar and pollen consumed by the bees per day, field residues do not induce the immediate death of the bees but rather chronic effects and damage (Cresswell 2011). It has been reported that field-dose neonicotinoid insecticides do not cause acute mortality in bees, but neonicotinoids can induce sublethal effects, such as a reduction in the number of synaptic units in the calyces of mushroom bodies (Peng and Yang 2016). Decreased bee immunity, diminished resistance to disease (Brandt et al. 2016), and reduced learning and memory are also consequences of neonicotinoid pesticide field residues (Aliouane et al. 2009; Alkassab and Kirchner 2016; Mengoni Gonalons and Farina 2015). Additionally, the use of neonicotinoid insecticides significantly reduces the foraging and homing capacity of collected bees (Fischer et al. 2014; Henry et al. 2012; Tison et al. 2016) and affects the hygiene behavior of bee colonies (Tsvetkov et al. 2017).

At present, the effects of pesticides, including neonicotinoids, on the health and physiology of bees are mainly evaluated by research on single pesticides. However, a broader spectrum of pesticides is widely used to protect crops during agricultural production, and the practice might include formulated mixtures and pesticides in the field. In fact, bees are exposed to more than 150 types of pesticides in their natural environments (Biddinger et al. 2013; Thompson et al. 2014), as demonstrated by the detection of a number of germicides, herbicides, and insecticides in honeybee beehives (Kaya et al. 2015). The toxicity of the neonicotinoids thiamethoxam and clothianidin is greatly enhanced in honeybees after the long-term application of field doses of some fungicides (Tsvetkov et al. 2017). The difference between the observed and predicted effect concentrations was rarely more than tenfold, and $95 \%$ of 69 mixtures of pesticides that exerted synergistic effects included cholinesterase inhibitors or azole fungicides (Cedergreen 2014). Other studies have shown that the long-term exposure of bumblebees to two insecticides (neonicotinoids and pyrethroids) at concentrations close to field levels can impair their natural foraging behavior and increase worker mortality, and these effects result in significant reductions in hatching development and population success. Moreover, the foraging performance of worker bees, particularly the efficiency of pollen collection, was significantly reduced (Gill et al. 2012). Because bees are subjected to a variety of pesticides in their natural environment, it is necessary to understand the synergistic effects of pesticides on bee health and physiology. Insecticidefungicide synergy is well-established (Raimets et al. 2018), but insecticide-insecticide synergy has not previously been widely established.

In this study, we investigated the mortality of a mixture of thiamethoxam (Th) with one of the three commonly used insecticides, namely, $\lambda$ cyhalothrin $(\lambda$-cy), $\beta$-cypermethrin $(\beta$-cy), and abamectin, and we then examined the effects of these insecticides on the activity and expression of acetylcholinesterase (AChE), glutathione-S transferases (GSTs), polyphenol oxidase (PPO), and alkaline phosphatase (ALP), which are typical enzymes involved in metabolism and detoxication processes in honeybees. Our work aimed to provide a theoretical basis for further research on the effects of mixtures of insecticides on the physical health of honeybees and to provide guidance for the selection of pesticide combinations with respect to the protection of honeybee populations.

\section{MATERIAL AND METHODS}

\subsection{Sample collection}

Healthy honeybee (Apis mellifera ligustica) colonies were maintained in the apiary at the 
Apicultural Research Institute, Anhui Agricultural University, Anhui, China. The population had not previously been exposed to any pesticide. We selected three healthy bee colonies, chose frames with freshly capped worker broods and moved them into an incubator with constant temperature and humidity $\left(35{ }^{\circ} \mathrm{C}, \mathrm{RH} 60 \%\right.$, darkness). The newly emerged worker bees (within $12 \mathrm{~h}$ ) were obtained.

\subsection{Acute (48-hour) oral toxicity $\left(\mathrm{LD}_{50}\right)$ test}

The worker bees were divided into 24 groups for different treatments. Each group consisted of 30 1-day-old worker bees caged in a plastic box $(12 \times$ $10 \times 8 \mathrm{~cm})$ and fed in an incubator $\left(28^{\circ} \mathrm{C}, \mathrm{RH}\right.$ $60 \%$, darkness). The top of the plastic box was replaced with gauze, and a square gap was then cut on the side of the box. Two small holes were cut on the side of the bottom of the $10-\mathrm{mL}$ centrifuge tube (Biosharp, Hefei, China), and the tube was then filled with sucrose solution and laid flat in the bee cage for feeding. A gap was also cut on the other side to replace the pollen. During the first three days, the bees were regularly fed fresh sucrose solution $(50 \%, \mathrm{w} / \mathrm{w})$ and pollen. The pesticide treatments were conducted on day 4 after emergence. For the treatments, four toxicants, Th, $\lambda$-cy, $\beta$-cy, and $\mathrm{Ab}$ (analytically pure, Beijing Bellingway Technology Co., Ltd.), were used. All the original toxicants were dissolved in acetone to obtain stock solutions with a concentration of $1 \times$ $10^{6} \mu \mathrm{g} / \mathrm{L}$. We diluted the stock solution with $50 \%$ sucrose solution to obtain five gradient concentrations (Th 1000, 750, 400, 200, $100 \mu \mathrm{g} / \mathrm{L} ; \lambda$-cy 7.5 $\times 10^{4}, 6 \times 10^{4}, 4 \times 10^{4}, 2 \times 10^{4}, 1 \times 10^{4} \mu \mathrm{g} / \mathrm{L} ; \beta$-cy $2.5 \times 10^{4}, 1.5 \times 10^{4}, 1 \times 10^{4}, 5 \times 10^{3}, 2.5 \times 10^{3}$ $\mu \mathrm{g} / \mathrm{L}$; and $\mathrm{Ab} 100,50,25,15,10 \mu \mathrm{g} / \mathrm{L})$, The bees in the control group were fed a $50 \%$ sucrose solution and an equal amount of $0.03 \%$ solvent. All groups of bees were starved for $2 \mathrm{~h}$, and each group of bees was then fed one of the different solutions of sucrose with pesticides at different concentrations for $4 \mathrm{~h}$ using a feeding tube. The weight of these tubes was measured before and after feeding to determine the average amount of insecticides consumed by each bee, and the feed intake of each group of bees was calculated and recorded. Fortyeight hours after the pesticide-containing syrup was removed, the number of deaths in each treatment group was counted. The data provided statistically significant information only if the mortality of the control group was less than $5 \%$.

\subsection{Exposure of honeybees to sublethal doses of insecticides}

We prepared a single pesticide-sucrose solution and binary mixtures of thiamethoxam with one of the three other insecticides for the sublethal dose treatments. All four toxicants were dissolved in acetone to obtain stock solutions with concentrations of $1 \times 10^{6} \mu \mathrm{g} / \mathrm{L}$ and then diluted with $50 \%$ sucrose solution to the following solutions: 300 $\mu \mathrm{g} / \mathrm{L}$ Th, $2.64 \times 10^{4} \mu \mathrm{g} / \mathrm{L} \lambda$-cy, $1.04 \times 10^{4} \mu \mathrm{g} / \mathrm{L}$ $\beta$-cy, $33 \mu \mathrm{g} / \mathrm{L} \mathrm{Ab}, 300 \mu \mathrm{g} / \mathrm{L} \mathrm{Th}+2.64 \times 10^{4} \mu \mathrm{g} / \mathrm{L}$ $\lambda$-cy, $300 \mu \mathrm{g} / \mathrm{L} \mathrm{Th}+1.04 \times 10^{4} \mu \mathrm{g} / \mathrm{L} \beta$-cy, and $300 \mu \mathrm{g} / \mathrm{L} \mathrm{Th}+33 \mu \mathrm{g} / \mathrm{L}$ Ab. All the solutions contained a final volume ratio of acetone of $0.03 \%$, and $50 \%$ sucrose solution containing $0.03 \%$ acetone was used as the control. The experiment included seven treatment groups and one control group and three replicates of each group. Twenty-five 4-day-old bees from each replicate were starved for $2 \mathrm{~h}$, and each group of bees was fed $250 \mu \mathrm{L}$ of the corresponding contaminated $50 \%$ sugar water and sugar water for $4 \mathrm{~h}$ through feeding tubes. The dose of each insecticide fed to each bee was equal to the $\mathrm{LD}_{10}$ determined in the acute oral test. The bees were then switched to $50 \%$ sugar water for $48 \mathrm{~h}$, the number of deaths in each group of bees was counted, and the live bees in each group were divided into two batches.

\subsection{Detection of enzymatic activity}

We dissected different tissues (heads or abdomens) to extract protein as an enzyme solution, and the protein content of each solution was then quantified using a BCA quantification kit (Beyotime, Shanghai, China). The activities of AChE, GSTs, ALP, and PPO were tested using corresponding kits purchased from Nanjing Jiancheng Bioengineering Institute (Nanjing, China). Due to the differential expression of these enzymes, five bee heads from each replicate were pooled to measure the activities of AChE, and three abdomens were used to measure the GST, 
ALP, and PPO activities. The preparation of the enzyme solution, protein quantification, and enzymatic activity assays were performed according to the manufacturer's handbook.

\subsection{RNA extraction, cDNA synthesis, and qRT-PCR analysis}

The brain (with the hypopharyngeal glands removed) and midgut were collected, dissected on ice, and homogenized using an electric grinder. Ten brains and five midguts from each replicate were pooled for total RNA extraction. The AChE2 gene in the RNA samples extracted from the brain was detected, whereas the GST, PPO, and $A L P$ genes in the RNA samples extracted from the midguts were detected. cDNA synthesis was performed using the TRIzol reagent (Tiangen, Beijing, China) and a first-strand cDNA synthesis kit (Toyobo, Ōsaka, Japan) according to the manufacturer's instructions. Real-time quantitative PCRs were performed in triplicate using a StepOne Plus Real-time PCR instrument (Life Technologies, CA, USA) and a SYBR Green Mix Ex Taq kit (Toyobo). We adopted a two-step method for the amplification process: 40 cycles of denaturation for $10 \mathrm{sec}$ at $95^{\circ} \mathrm{C}$ and extension for $30 \mathrm{sec}$ at $60^{\circ} \mathrm{C}$. Rps 5 was used as the reference gene in the qRT-PCR assay, and relative gene expression was calculated using the $2^{-\Delta \Delta C t}$ method. The primers used for the qRT-PCR assay are shown in Table III.

\subsection{Statistical analysis}

All statistical analyses were performed using SPSS.24 software. The level of significance in all the tests was set to $P<0.05$. Probit regression was used to calculate the $\mathrm{LD}_{50}$ and $\mathrm{LD}_{10}$ values and the 95\% confidence intervals of the four toxicants on honeybees, and one-way ANOVA was used to assess the differences in enzyme activity and gene expression between the bees in each group. The significance of the differences was tested using Duncan's method. The synergistic effect of thiamethoxam with the three other insecticides was assessed on bee toxicity using the "modified binomial proportion" recommended by Sgolastra et al. (2016). All the graphs shown in this manuscript were prepared using GraphPad Prism 5 software.

\section{RESULTS}

\subsection{Determination of the toxicity of four insecticides on honeybees}

The values of the LD50 (ng/bee) and the LD10 (ng/bee) for each insecticide are presented in the Table I. These data clearly showed among the four tested insecticides, $\mathrm{Ab}$ was the most lethal to honeybees, whereas $\lambda$-cy clearly showed the least toxicity.

\subsection{Effects of insecticides on survival of honeybees}

In practice, a mixture of insecticides is usually used to achieve better effects in pest control. Therefore, we subsequently focused on determining the combined effects of a mixture of Th with one of the three other insecticides. We prepared binary mixtures based on the $\mathrm{LD}_{10}$ concentrations obtained from the acute toxicity data. As shown in Figure 1, the mortality rates of the bees in each insecticide treatment group were significantly higher than that of the control group $(P<0.05)$, and the highest mortality rate, which reached $44.4 \%$, was obtained with the $\mathrm{Th}+\mathrm{Ab}$ mixture, which was consistent with the results shown in Table I. In addition, the mortality rates of the groups treated with the $\mathrm{Th}+\lambda$-cy and $\mathrm{Th}+\mathrm{Ab}$ mixtures were significantly increased compared with those of the three single-treatment groups, which suggested that these two combinations exhibited enhanced toxicity.

We also assessed the synergistic effects of thiamethoxam with the three other insecticides on bee toxicity using the "modified binomial proportion" recommended by Sgolastra et al. (2016). The Th $+\lambda$-cy $\left(\chi^{2}=4.65, P<0.05\right)$ and $\mathrm{Th}+\mathrm{Ab}$ $\left(\chi^{2}=9.96, P<0.01\right)$ combinations exerted synergistic effects on bee toxicity, whereas the Th + $\beta$-cy mixture did not exert a synergistic effect $\left(\chi^{2}\right.$ $=0.53, P>0.05$; Table II).

\subsection{Effects of insecticides on enzyme activity in honeybees}

To better understand the molecular basis of the effects of the mixtures, we tested the PPO, ALP, 
Table I. Oral toxicity of four insecticides to honeybees

\begin{tabular}{lllll}
\hline Insecticides & Time $(\mathrm{h})$ & Regression equation & $\mathrm{LD}_{50}(\mathrm{ng} / \mathrm{bee})(95 \% \mathrm{CL})$ & $\mathrm{LD}_{10}(\mathrm{ng} / \mathrm{bee})$ \\
\hline $\mathrm{Th}$ & 48 & $\mathrm{y}=2.68 \times-2.56$ & $9.02(5.87 \sim 13.04)$ & 3 \\
$\lambda$-cy & 48 & $\mathrm{y}=2.52 \times-7.38$ & $854.63(546.64 \sim 1270.59)$ & 264.46 \\
$\beta$-cy & 48 & $\mathrm{y}=3.02 \times-7.34$ & $277.28(197.98 \sim 406.86)$ & 104.26 \\
$\mathrm{Ab}$ & 48 & $\mathrm{y}=3.33 \times-0.34$ & $0.79(0.58 \sim 1.13)$ & 0.33 \\
\hline
\end{tabular}

and GST enzyme activities in the midgut and the AChE activity in the brain after pesticide treatment.

As shown in Figure 2a, the PPO activity of the bees was significantly increased after treatment with Th, $\lambda$-cy, or Ab alone $(P<0.05)$. However, the activity of PPO in the $\beta$-cy-treated group was equal to that of the control group and significantly lower than that of the other treatment groups $(P<$ $0.05)$. Interestingly, the groups treated with the $\mathrm{Th}$ $+\lambda$-cy and $\mathrm{Th}+\mathrm{Ab}$ combinations showed clearly decreased PPO activity compared with the groups administered the corresponding single-pesticide treatments $(P<0.05)$. In contrast, the Th $+\beta$-cy combination had an intermediate effect on PPO activity.

The AChE activity of the bees treated with Th or $\lambda$-cy was higher than that of the other treatment groups $(P<0.05)$. The enzyme activities of the groups treated with the binary mixtures were significantly lower than those of the group treated with Th alone but showed no significant difference $(P<0.05)$ compared with the control group (Figure 2b).

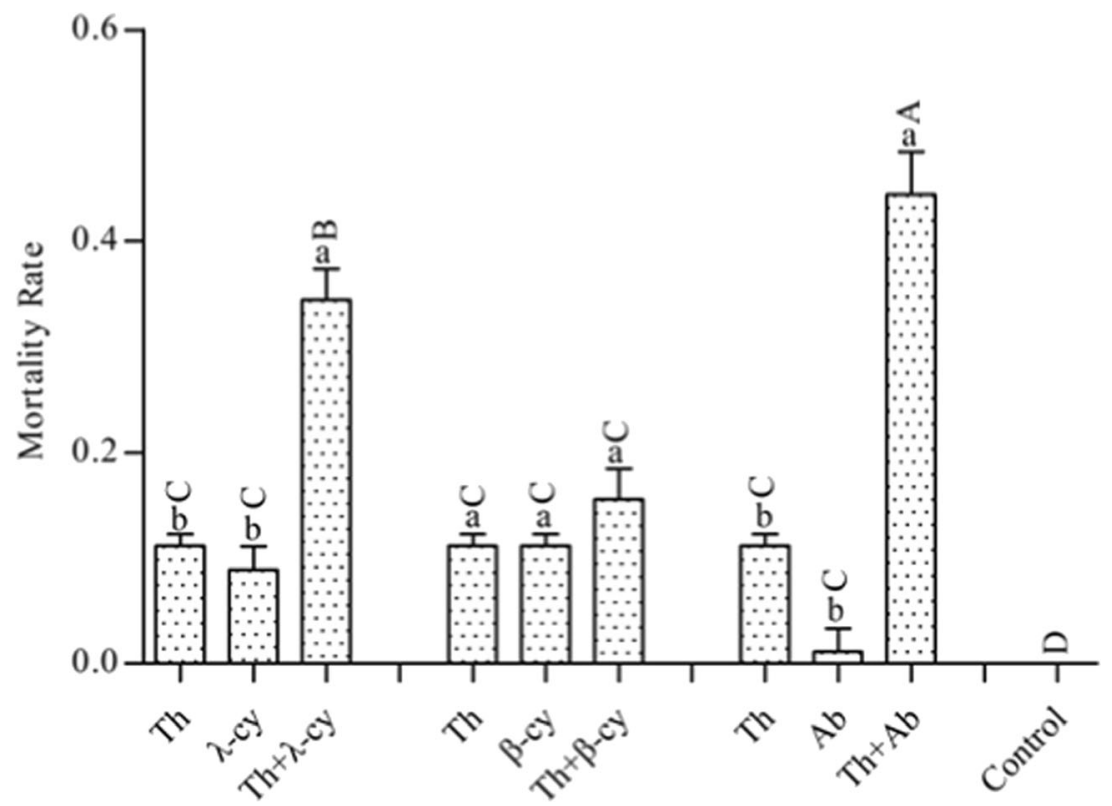

Figure 1. Effect of thiamethoxam and three insecticides administered as single and mixed treatments on the survival of Apis mellifera. The data in the graph are shown as the means \pm standard errors. Different capital letters indicate a significant difference $(P<0.05)$ among all the treatment groups, and different lowercase letters indicate a significant difference between the single- and mixed-pesticide treatments $(P<0.05)$. This information also applies to the rest of the figures. $\mathrm{X}$-axis, pesticide treatment; $\mathrm{y}$-axis, mortality rate. Th thiamethoxam, $\lambda$-cy lambda-cyhalothrin, $\beta$-cy beta-cypermethrin, $A b$ abamectin. 
Table II. Mortality of thiamethoxam and three pesticides administered alone and in a mixture on honeybees

\begin{tabular}{lllll}
\hline Pesticide & Number of bees & Mortality Rate \pm SE $(\%)$ & $\chi^{2}$ & $P$-value \\
\hline Th & 75 & $11.11 \pm 0.01$ & \\
$\lambda$-cy & 75 & $8.88 \pm 0.02$ & \\
$\beta$-cy & 75 & $11.11 \pm 0.01$ & & \\
Ab & 75 & $11.11 \pm 0.02$ & & \\
Th $+\lambda$-cy & 75 & $34.44 \pm 0.03$ & 4.65 & $P<0.05$ \\
Th $+\beta$-cy & 75 & $15.56 \pm 0.03$ & 0.53 & $P>0.05$ \\
Th + Ab & 75 & $44.44 \pm 0.04$ & 9.96 & $P<0.01$ \\
\hline
\end{tabular}

The activity of GSTs after Th treatment was similar to that of the control group (Figure 2c), which was consistent with previous studies (Tavares et al. 2017). Similar results were found in the groups administered the $\beta$-cy and $\mathrm{Ab}$ single treatments, but the administration of $\beta$-cy decreased the activity of GSTs. Moreover, the GST activities of the three groups treated with the binary mixtures were significantly lower than those of the control group $(P<0.05)$.

The activity of ALP was affected by all the treatments. Specifically, ALP activity was reduced after all the pesticide treatments, particularly the $\mathrm{Th}+\mathrm{Ab}$ and $\mathrm{Th}+\beta$-cy mixtures.

\section{4. mRNA expression level of enzymes after administration of the four insecticides}

To further assay whether insecticides affect the mRNA expression of enzymes, the mRNA levels of the four enzymes after administration of the four tested insecticides were tested, and the results showed that the insecticides significantly affected the mRNA expression levels of the four enzymes (Figure 3). Compared with the control group, the expression level of all four enzymes was affected by the administration of Th alone; specifically, Th alone increased the expression of $A C h E 2$ and decreased the expression of the other three enzymes. The single administration of $\lambda$-cy reduced the expression level of $P P O$, increased the expression level of $A C h E 2$ and GST, and did not affect the expression level of $A L P$. The expression level of $A L P$ was slightly elevated by the administration of $\beta$-cy alone. However, the single administration of $\mathrm{Ab}$, which was the most toxic pesticide among those evaluated in this study, did not disturb the gene expression of the four enzymes.

As shown in Figure 3a-3c, the gene expression of $P P O$ and GST was significantly reduced by the

Table III. Primers for qRT-PCR

\begin{tabular}{llll}
\hline Gene name & Sequence & Annealing temperature & Gene ID \\
\hline$P P O$ & F: 5'-GGAGGTGATAGTTTGGAACGA-3' & 53 & GB43738 \\
R: 5'-TTGAGGCATCCTTACAACCA-3' & & GB41856 \\
AChE2 & F: 5'-GACGCGAAGACCATATCCGT-3' & 56 & \multirow{2}{*}{ GB48904 } \\
GST & R: 5'-TCTGTGTCCTTGAAGTCCGC-3' & \multirow{2}{*}{55} & GB52063 \\
\multirow{2}{*}{ ALP } & F: 5'-TGCATATGCTGGCATTGATT-3' & & \\
\multirow{2}{*}{ RpS5 } & R: 5'-TCCTCGCCAAGTATCTTGCT-3' & 53 & GB11132 \\
& F: 5'-GATTCCATTCAGAGACGAATGC-3' & & \\
\hline & R: 5'-GCATTCTCCAGTAGATCTTTCAG-3' & \multirow{2}{*}{56} &
\end{tabular}



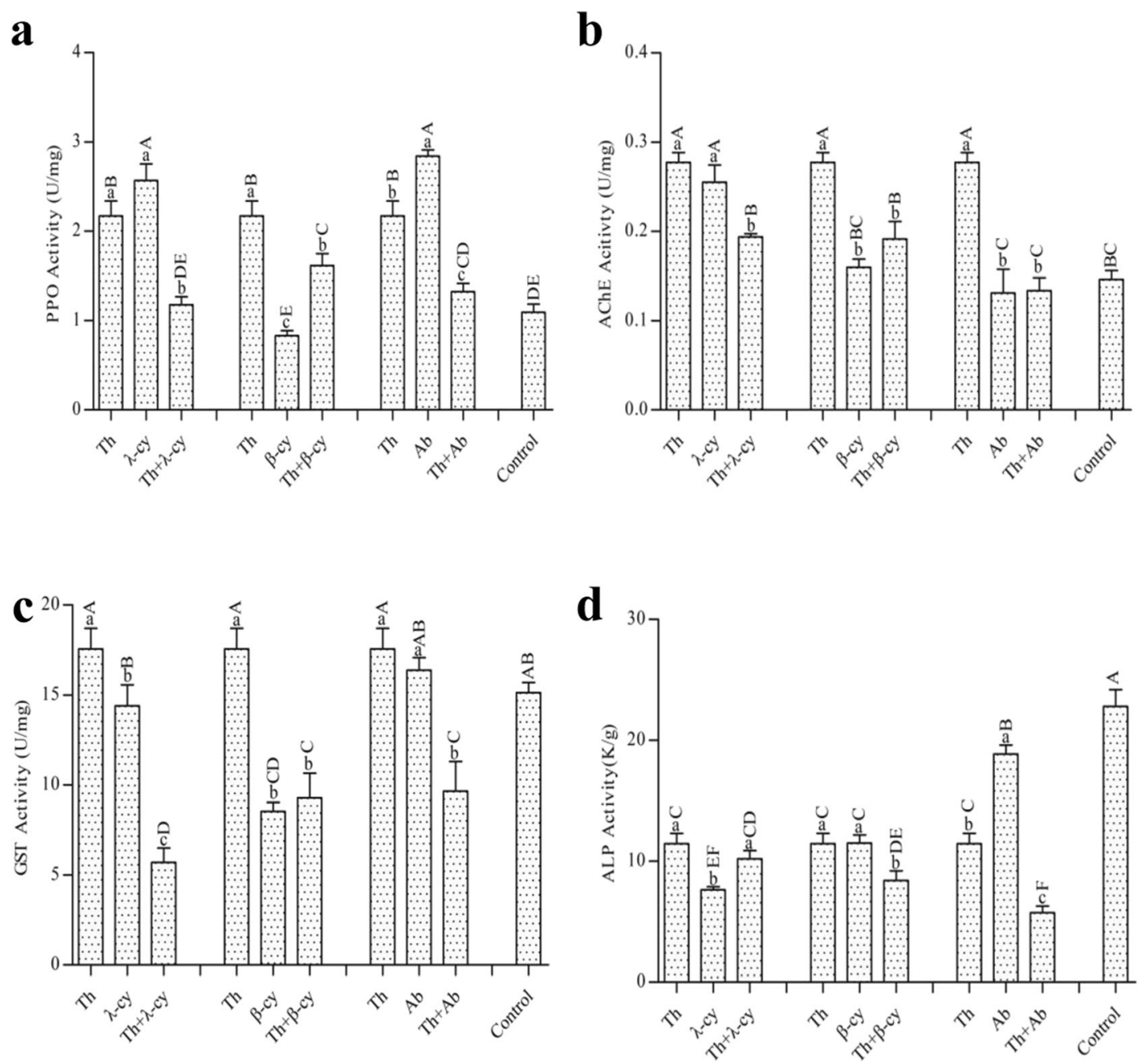

Figure 2. Effects of insecticides on enzyme activity in Apis mellifera. The activities of the four indicated enzymes after different pesticide treatments were tested. X-axis, pesticide treatment; y-axis, enzyme activity (U/mg for PPO/ AChE/GSTs; K/g for ALP). Th thiamethoxam, $\lambda$-cy lambda-cyhalothrin, $\beta$-cy beta-cypermethrin, $A b$ abamectin, $P P O$ polyphenol oxidase, $A C h E$ acetylcholinesterase, GSTS glutathione S-transferases, $A L P$, alkaline phosphatase.

three binary mixture treatments, but none of these treatments affected the expression of AChE2. Specifically, $A L P$ expression was weakly reduced after treatment with $\mathrm{Th}+\lambda$-cy and was not affected by $\mathrm{Th}+\beta$-cy. In contrast, the gene expression of $A L P$ in honeybees was elevated up to twofold after treatment with the $\mathrm{Th}+\mathrm{Ab}$ combination relative to the control.

\section{DISCUSSION}

Our findings indicated that the $\mathrm{Th}+\lambda$-cy and $\mathrm{Th}+\mathrm{Ab}$ combinations could induce synergistically increased toxicity, as demonstrated by the finding that the mortality in the groups exposed to both insecticides is greater than the sum of the mortalities induced by each insecticide taken separately. This result is similar to that obtained for the neonicotinoid pesticide imidacloprid, and mixtures with $\lambda$-cy significantly increase the mortality of bees (Zhu et al. 2017). Furthermore, the highest bee mortality was obtained with the $\mathrm{Th}+\mathrm{Ab}$ treatment, and this treatment resulted in significantly higher mortality compared with the other treatments. However, no significant difference in bee mortality was 
$\mathbf{a}$

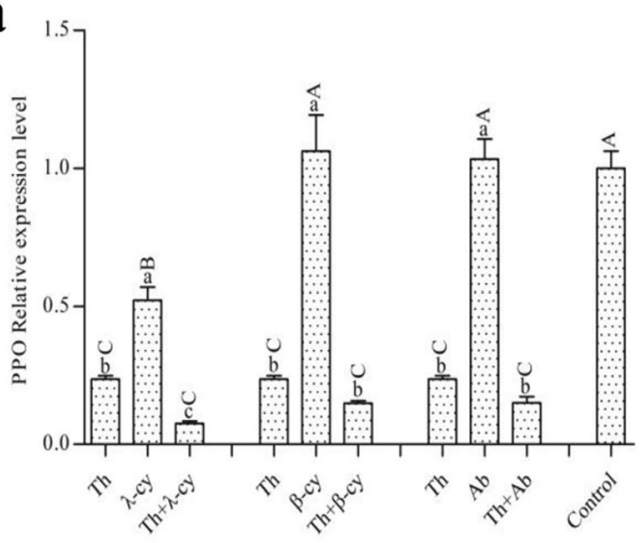

c

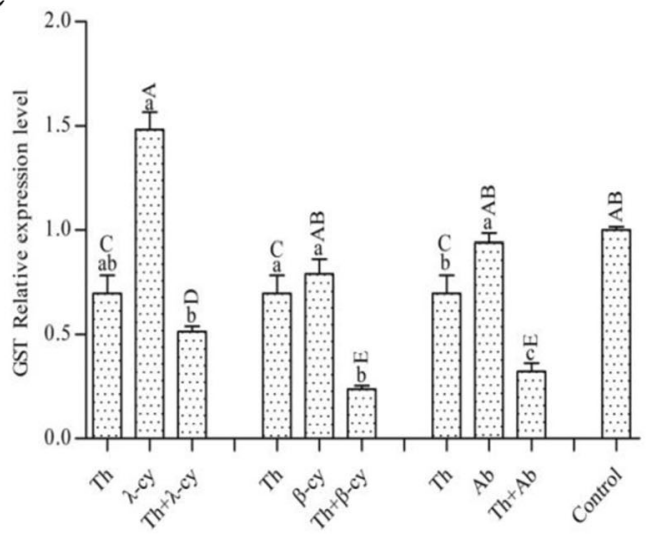

b

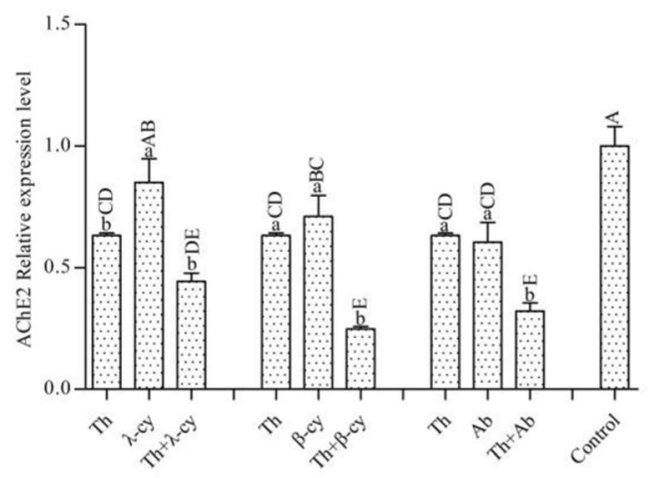

d

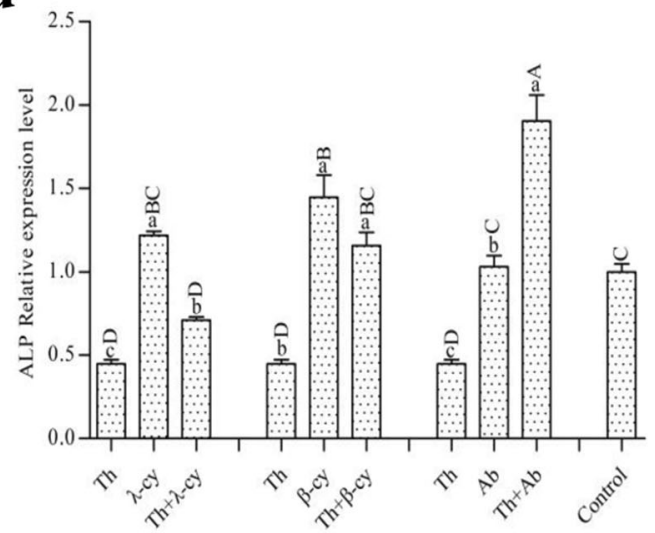

Figure 3. Relative mRNA expression of enzymes. The relative mRNA expression levels of four enzymes after the different pesticide treatments were tested by qRT-PCR. X-axis, pesticide treatment; y-axis, relative mRNA expression level. Th thiamethoxam, $\lambda$-cy lambda-cyhalothrin, $\beta$-cy beta-cypermethrin, $A b$ abamectin, $P P O$ polyphenol oxidase, $A C h E$ acetylcholinesterase, GSTs glutathione S-transferases, $A L P$ alkaline phosphatase.

found between the Th $+\beta$-cy mixture and the single-treatment groups, which suggested that the $\mathrm{Th}+\lambda$-cy and $\mathrm{Th}+\mathrm{Ab}$ binary mixtures exerted more adverse effects than the individual insecticides. Thus, these combinations are more lethal to bee colonies. Nevertheless, the doses of the single treatments were based on the $\mathrm{LD}_{10}$ data and were slightly higher than the doses used in the field. Whether the use of different doses would change the effects will be investigated in a future study.

The pharmacological mechanisms of individual insecticides have been thoroughly studied, but the toxic effects of pesticide mixtures on organisms are frequently unclear. The use of enzyme activities as an indicator in toxicology studies could improve our understanding of the toxic effects caused by mixtures of insecticides. The exposure of bees to insecticides rapidly activates signal pathways related to detoxification and metabolism to eliminate or minimize the effects of the toxins in vivo, and during this exposure, the activity of relevant enzymes shows changes to varying extents (Boily et al. 2013; Li et al. 2017). The treatment of bees with different pesticides alone exerts various effects on enzymes, and these effects depend on the mechanisms of the insecticides and the properties of the enzymes. Numerous factors, including the cell status, substrate concentration, enzyme structure, cofactor 
levels, and changes in enzyme expression, affect enzyme activity. In our study, we assayed various enzyme activities and various mRNA expression levels after treatment with the binary mixtures of insecticides to provide initial insights into this reaction. Our subsequent in vitro examinations of the activities of one detoxification enzyme (GST), one insecticide-target enzyme (AChE), and two immunity enzymes (PPO and ALP) in the bees that survived after treatment with thiamethoxam or the various mixtures provided substantial additional knowledge on the impact of insecticides on honeybee physiology. In insects, PPO is an important innate immune protein that is involved in cellular and humoral defenses. Exposure of a host to exogenous substances rapidly activates PPO in vivo to protect it from infection (Hillyer et al. 2003; James and Xu 2012). AChE mainly controls the neural activity of cholinergic synapses (Badiou et al. 2008) and can rapidly hydrolyze acetylcholine (Ach) in the insect's central nervous system, thereby terminating the excitotoxic effects of Ach on the postsynaptic membrane and ensuring the normal transmission of nerve signals (Casida and Durkin 2013). Glutathione S-transferase is an important detoxification enzyme in honeybees that reflects the antioxidant stress of bees. The GSTO1 family is vital for protecting the body from oxidative damage (Jia et al. 2014). The bee GSTs which are the major detoxification enzymes in the second detoxification phase catalyze the breakdown of metabolic toxins produced by the first-stage detoxification enzymes, such as cytochrome P450 monooxygenase and carboxylesterase (Berenbaum and Johnson 2015). GST activity could be enhanced by flumethrin and is thought to be involved in the detoxification and metabolism of certain toxins by bees (Nielsen et al. 2000). ALP is a type of nonspecific phosphate hydrolytic enzyme that can hydrolyze and transfer phosphate groups. This highly conserved enzyme family, which is directly involved in the transfer and metabolism of phosphate groups in organisms, is generally regarded as a hydrolytic enzyme related to insect resistance (Lalles 2010; Millan 2006). ALP is mainly associated with the hydrolysis of phosphate groups in different substrates, and this reaction is involved in substance absorption, intestinal integrity and homeostasis, and immune processes (Zibaee et al. 2011). The effect of the Th $+\mathrm{Ab}$ mixture, which was the most toxic combination, on PPO and AChE activities was not clearly different from that of the control group but significantly repressed the activity of GST and ALP. Based on this result, we hypothesized that the $\mathrm{Th}+\mathrm{Ab}$ combination disturbed the normal function of GSTs and ALP and aggravated the damage to detoxification and the immune system, which resulted in the highest mortality rate. The analysis of the mRNA expression levels showed that this treatment exerted distinct effects on the expression of all four enzymes. As mentioned above, alterations in enzyme expression levels are one of the reasons for the observed changes in enzyme activity. Changes in enzyme activities might be a passive process caused by deterioration of the cell microenvironment, which explains the seemingly irregular correlation between enzyme activity and expression changes. Therefore, the complexity of the effects of pesticides on organisms appears more apparent. In contrast, changes in enzyme activity are rapid and dynamic processes that are accurately regulated, and our data provide basic or probable evidence explaining the elevation in the mortality rate obtained with the binary mixtures; however, this approach was not sufficient for clearly solving this problem. In future research, we will consider the dose and time effects of the insecticide mixtures and will study the effects of the insecticide mixtures in more detail.

\section{CONCLUSION}

In summary, the combination of a lethal dose of Th with $\lambda$-cy or Ab showed synergistically increased toxicity to honeybees, but the $\mathrm{Th}+\beta$-cy mixture did not significantly affect bee mortality. Exposure of the bees to the stress induced by administration of the insecticides alone or in mixtures also changed the activities of all of the four enzymes studied here. The results suggest that the $\mathrm{Th}+\lambda$-cy and $\mathrm{Th}+\mathrm{Ab}$ combinations at a known dose will adversely affect bee survival, which raises the risk for apiculture. Taken together, our findings provide a theoretical basis for the indepth study of the effects of insecticide mixtures 
on the health and physiology of honeybees. The results also have a certain guiding significance for the rational application of insecticides for bee protection in agricultural production.

\section{ACKNOWLEDGMENTS}

This work was financially supported by the National Special Construction Apiculture Technology System of China (No. CARS-45-KXJ10). We are grateful to Prof Jianke Li for reviewing this manuscript.

\section{AUTHORS' CONTRIBUTIONS}

All the authors conceived and designed the experiments; Yufei Wang performed the experiments and analyses; Yufei Wang and Wenxin Zhang wrote the paper; and all the authors helped revised the manuscript and approved the final manuscript.

Toxicité synergique et impact physiologique sur les abeilles mellifères du thiaméthoxame seul ou en mélange binaire avec trois insecticides d'usage courant.

abeille mellifère / insecticides / thiaméthoxame / mélange binaire / effet synergique.

Synergistische Toxizität und physiologischer Einfluss auf Honigbienen von Thiamethoxam allein oder in Mischung mit jeweils einem von drei häufig genutzten Insektiziden.

Honigbiene / Insektizide / Thiamethoxam / Mischung aus zwei Komponenten / synergistischer Effekt.

\section{REFERENCES}

Aliouane, Y., El Hassani, A.K., Gary, V., Armengaud, C., Lambin, M., and Gauthier, M. (2009) Subchronic exposure of honeybees to sublethal doses of pesticides: effects on behavior. Environ Toxicol Chem. 28(1), 113-122

Alkassab, A.T., and Kirchner, W.H. (2016) Impacts of chronic sublethal exposure to clothianidin on winter honeybees. Ecotoxicology. 25(5), 1000-1010

Badiou, A., Meled, M., and Belzunces, L.P. (2008) Honeybee Apis mellifera acetylcholinesterase-a biomarker to detect deltamethrin exposure. Ecotoxicol Environ Saf . 69(2), 246-253
Berenbaum, M.R., and Johnson, R.M. (2015) Xenobiotic detoxification pathways in honey bees. Curr Opin Insect Sci. 10, 51-58

Biddinger, D.J., Robertson, J.L., Mullin, C., Frazier, J., Ashcraft, S.A., Rajotte, E.G., Joshi, N.K., and Vaughn, M. (2013) Comparative toxicities and synergism of apple orchard pesticides to Apis mellifera (L.) and Osmia cornifrons (Radoszkowski). PLoS One. 8(9), e72587

Boily, M., Sarrasin, B., Deblois, C., Aras, P., and Chagnon, M. (2013) Acetylcholinesterase in honey bees (Apis mellifera) exposed to neonicotinoids, atrazine and glyphosate: laboratory and field experiments. Environ Sci Pollut Res Int. 20(8), 5603-5614

Brandt, A., Gorenflo, A., Siede, R., Meixner, M., and Buchler, R. (2016) The neonicotinoids thiacloprid, imidacloprid, and clothianidin affect the immunocompetence of honey bees (Apis mellifera L.). J Insect Physiol. 86, 40-47

Casida, J.E., and Durkin, K.A. (2013) Neuroactive insecticides: targets, selectivity, resistance, and secondary effects. Annu Rev Entomol. 58(1), 99-117

Cedergreen, N. (2014) Quantifying synergy: a systematic review of mixture toxicity studies within environmental toxicology. PLOS ONE. 9(5):e96580

Christen, V., Joho, Y., Vogel, M., and Fent, K. (2018) Transcriptional and physiological effects of the pyrethroid deltamethrin and the organophosphate dimethoate in the brain of honey bees (Apis mellifera). Environmental Pollution.244, 247-256

Cresswell, J.E. (2011) A meta-analysis of experiments testing the effects of a neonicotinoid insecticide (imidacloprid) on honey bees. Ecotoxicology. 20(1), $149-157$

Elbert, A., Haas, M., Springer, B., Thielert, W., and Nauen, R. (2008) Applied aspects of neonicotinoid uses in crop protection. Pest Manag Sci. 64(11), 1099-1105

Fischer, J., Muller, T., Spatz, A.K., Greggers, U., Grunewald, B., and Menzel, R. (2014) Neonicotinoids interfere with specific components of navigation in honeybees. PLoS One. 9, e91364

Gill, R. J., Ramos-Rodriguez, O., \& Raine, N. E. (2012) Combined pesticide exposure severely affects individual- and colony-level traits in bees. Nature. 491(7422):105-108

Goulson, D., Nicholls, E., Botias, C., and Rotheray, E.L. (2015) Bee declines driven by combined stress from parasites, pesticides, and lack of flowers. Science. 48(3), 328-339

Henry, M., Beguin, M., Requier, F., Rollin, O., Odoux, J.F., Aupinel, P., Aptel, J., Tchamitchian, S., and Decourtye, A. (2012) A common pesticide decreases foraging success and survival in honey bees. Science. 336(6101), 348-350

Hillyer, J.F., Schmidt, S.L., and Christensen, B.M. (2003) Hemocyte-mediated phagocytosis and melanization in the mosquito Armigeres subalbatus following immune challenge by bacteria. Cell Tissue Res. 313(1), 117127 
James, R.R., and Xu, J. (2012) Mechanisms by which pesticides affect insect immunity. J Invertebr Pathol. 109(2), 175-182

Jia, H., Sun, R., Shi, W., Yan, Y., Li, H., Guo, X., and Xu, B. (2014) Characterization of a mitochondrial manganese superoxide dismutase gene from Apis cerana cerana and its role in oxidative stress. J Insect Physiol. 60(1), 68-79

Kaya, M., Erdogan, S., Mol, A., and Baran, T. (2015) Comparison of chitin structures isolated from seven Orthoptera species. Int J Biol Macromol 72, 797-805

Lalles, J.P. (2010) Intestinal alkaline phosphatase: multiple biological roles in maintenance of intestinal homeostasis and modulation by diet. Nutr Rev. 68(6), 323-332

Li, Z., Li, M., He, J., Zhao, X., Chaimanee, V., Huang, W.F., Nie, H., Zhao, Y., and Su, S. (2017) Differential physiological effects of neonicotinoid insecticides on honey bees: a comparison between Apis mellifera and Apis cerana. Pestic Biochem Physiol. 140, 1-8

Liao, C.H., He, X.J., Wang, Z.L., Barron, A.B., Zhang, B., Zeng, Z.J., and Wu, X.B. (2018) Short-term exposure to lambda-cyhalothrin negatively affects the survival and memory-related characteristics of worker bees Apis mellifera. Archives of Environmental Contamination and Toxicology. 75(1): 59-65

Matsuda, K., Buckingham, S.D., Kleier, D., Rauh, J.J., Grauso, M., and Sattelle, D.B. (2001) Neonicotinoids: insecticides acting on insect nicotinic acetylcholine receptors. Trends Pharmacol Sci. 22(11), 573-580

Mengoni Gonalons, C., and Farina, W.M. (2015) Effects of sublethal doses of imidacloprid on young adult honeybee behaviour. PLoS One. 10(10), e0140814

Millan, J.L. (2006) Alkaline phosphatases: structure, substrate specificity and functional relatedness to other members of a large superfamily of enzymes. Purinergic Signal. 2(2), 335-341

Millar, N.S., and Denholm, I. (2007) Nicotinic acetylcholine receptors: targets for commercially important insecticides. Invert Neurosci. 7(1), 53-66

Moritz, R.F.A., de Miranda, J., Fries, I., Le Conte, Y., Neumann, P., and Paxton, R.J. (2010) Research strategies to improve honeybee health in Europe. Apidologie. 41(3), 227-42

Nazzi, F., and Le Conte, Y. (2016) Ecology of Varroa destructor, the major ectoparasite of the Western honey bee,Apis mellifera Annu Rev Entomol. 61, 417-32

Nielsen, S.A., Brodsgaard, C.J., and Hansen, H. (2000) Effects on detoxification enzymes in different life stages of honey bees (Apis mellifera L., Hymenoptera: Apidae) treated with a synthetic pyrethroid (flumethrin). Altern Lab Anim. 28(3), 437-443
Peng, Y. C. , \& Yang, E. C. (2016). Sublethal dosage of imidacloprid reduces the microglomerular density of honey bee mushroom bodies. Scientific Reports, 6(1), 19298.

Raimets, R., Karise, R., Mänd, M., Kaart, T., Ponting, S., Song, J., Cresswell, J.E. (2018) Synergistic interactions between a variety of insecticides and an ergosterol biosynthesis inhibitor fungicide in dietary exposures of bumble bees (Bombus terrestris L.). Pest Management Science, 74(3), 541-546

Sgolastra, F., Medrzycki, P., Bortolotti, L., Renzi, M. T., \& Bosch, J. (2016) Synergistic mortality between a neonicotinoid insecticide and an ergosterolbiosynthesis-inhibiting fungicide in three bee species. Pest Management Science, 73(6), 1236-1243

Tavares, D.A., Dussaubat, C., Kretzschmar, A., Carvalho, S.M., Silva-Zacarin, E.C.M., Malaspina, O., Berail, G., Brunet, J.L., and Belzunces, L.P. (2017) Exposure of larvae to thiamethoxam affects the survival and physiology of the honey bee at post-embryonic stages. Environ Pollut. 229, 386-393

Thompson, H.M., Fryday, S.L., Harkin, S., and Milner, S. (2014) Potential impacts of synergism in honeybees (Apis mellifera) of exposure to neonicotinoids and sprayed fungicides in crops. Apidologie. 45(5), 545553

Tison, L., Hahn, M.L., Holtz, S., Rossner, A., Greggers, U., Bischoff, G., and Menzel, R. (2016) Honey bees' behavior is impaired by chronic exposure to the neonicotinoid thiacloprid in the field. Environ Sci Technol. 50(13), 7218-7227

Tsvetkov, N., Samson-Robert, O., Sood, K., Patel, H.S., Malena, D.A., Gajiwala, P.H., Maciukiewicz, P., Fournier, V., and Zayed, A. (2017) Chronic exposure to neonicotinoids reduces honey bee health near corn crops. Science. 356(6345), 1395-1397

Zhu, Y.C., Yao, J.X., Adamczyk, J., and Luttrell, R. (2017) Synergistic toxicity and physiological impact of imidacloprid alone and binary mixtures with seven representative pesticides on honey bee (Apis mellifera ). Plos One 12(5), e0176387

Zibaee, A., Zibaee, I., and Sendi, J.J. (2011) A juvenile hormone analog, pyriproxyfen, affects some biochemical components in the hemolymph and fat bodies of Eurygaster integriceps Puton (Hemiptera: Scutelleridae). Pestic Biochem Phys 100, 289-298

Publisher's note Springer Nature remains neutral with regard to jurisdictional claims in published maps and institutional affiliations. 